\title{
CCD Spectrophotometry of CC Cas. I. Radial Velocity Curves
}

\author{
S. Yu. Gorda \\ Kourovka Astronomical Observatory, Ural Federal University, Ekaterinburg, 620083 Russia
}

Received October 1, 2012; in final form, November 6, 2012

\begin{abstract}
We report the results of the first-ever CCD spectroscopic observations of the eclipsing variable CC Cas with massive early-type components. The measured semi-amplitudes of the radial velocity curves of the components, $K_{1}=123.9 \mathrm{~km} / \mathrm{s}$ and $K_{2}=292.4 \mathrm{~km} / \mathrm{s}$, are comparable with the published data, and the center-of-mass velocity of the system was found to be $V_{0}=-20.4 \mathrm{~km} / \mathrm{s}$, which is about $-10 \mathrm{~km} / \mathrm{s}$ greater than the earlier published results. This fact may support the published hypothesis about the presence of a third body in the system.
\end{abstract}

DOI: $10.1134 / \mathrm{S} 1990341313010094$

Keywords: stars: binaries: eclipsing-stars: individual: CC Cas

\section{INTRODUCTION}

CC Cas-an eclipsing variable with massive earlytype components, O8.5 III + B0V $\left(V=7{ }^{\mathrm{m}} 08\right.$, $P=3$ d.366324)—was discovered by Pearce [1] in 1927 as a spectroscopic binary. In 1930 Guthnick and Prager [2] found the star to be photometrically variable, and Gaposhkin [3] confirmed its variability in 1953. Although various authors performed photoelectric observations of CC Cas in the 1960-1980s (see, e.g., [4]), only three light curves with a more or less complete phase coverage were obtained during this time. The shape of the light curve allows the star to be classified as EB. All of the obtained light curves show a large scatter of points as well as temporary distortions of the shape at some phase angles. Such a behavior indicates that CC Cas is an active interacting system, possibly with a strong stellar wind blowing off the surface of the primary.

Most of the spectroscopic observations of CC Cas starting from the first study by Pearce [1] were photographic. The spectrograms showed well-defined lines of the primary, whereas those of the secondary were discernible only on some spectra and were not measurable. In 1985-87 the first photoelectrically recorded (reticon) spectra of CC Cas were taken by Hill et al. [4] that allowed the lines of the secondary companion to be extracted and measured. The above authors obtained the radial velocity curves of both CC Cas components, inferred a credible estimate of the component mass ratio, and determined the evolutionary status of the system.

Some of the authors who determined the orbital parameters of the primary found the orbit to be elliptical with an eccentricity of about 0.1 [1]. Moreover, the ellipticity was most often found in the spectroscopic observations; it did not show up in photometric studies. Also notable is a certain discrepancy between the estimates of the period of CC Cas obtained by different authors at different times. The photometric determinations usually yielded a shorter period than the spectroscopic studies. Furthermore, in the early 70 s a significant change in the center-of-mass velocity of the system was observed [4]. See Hill et al. [4] and Mayer [5] for a more detailed discussion of the period change and a possible orbital ellipticity.

Although small changes in the period have been registered for a long time, their cause still remains unclear. Two possible effects are considered: (1) the loss of mass by the system due to the strong stellar wind detected at radio frequencies [6] and in the optical part of the spectrum [7] and (2) the presence of a third body in the system. The former possibility appears to be more likely, however, the latter also cannot be ruled out because of the detected variation of the systemic velocity. Hill et al. [4] pointed out that "... such an explanation (the presence of a third body, author's note) cannot yet be ruled out, and it would provide a model for the above velocity differences and some of the larger deviations of times of minima from the linear ephemeris." One way or another, this issue still remains open.

No spectroscopic studies of CC Cas have been performed since the mid-80s. It was therefore of interest to include this system in the program of spectroscopic observations with the echelle spectrometer of the new 1.2-m telescope of the Kourovka Astronomical Observatory of the Ural Federal University, especially since an earlier photometric study of the 
Table 1. List of the obtained spectra and the measured radial velocities of the components of CC Cas

\begin{tabular}{c|c|c|c|c|r|r|r|r}
\hline No. & Date & $\begin{array}{c}n \times t, \\
\mathrm{c}\end{array}$ & $\begin{array}{c}\mathrm{JD} \odot \\
2455000+\end{array}$ & Phase & $\begin{array}{r}V_{1}, \\
\mathrm{~km} / \mathrm{s}\end{array}$ & $\begin{array}{c}\sigma_{1}, \\
\mathrm{~km} / \mathrm{s}\end{array}$ & $\begin{array}{c}V_{2}, \\
\mathrm{~km} / \mathrm{s}\end{array}$ & $\begin{array}{c}\sigma_{2}, \\
\mathrm{~km} / \mathrm{s}\end{array}$ \\
\hline 1 & 30.03 .11 & $3 \times 1200$ & 651.1943 & 0.120 & -101.0 & 0.9 & 199.6 & 7.0 \\
2 & 24.11 .11 & $3 \times 1200$ & 889.5081 & 0.908 & 55.6 & 1.2 & -194.1 & 12.8 \\
3 & 24.11 .11 & $3 \times 1200$ & 889.5539 & 0.921 & 39.7 & 2.1 & -167.0 & 17.6 \\
4 & 24.11 .11 & $2 \times 1200$ & 889.5913 & 0.932 & 34.3 & 1.8 & -168.1 & 11.9 \\
5 & 18.01 .12 & $3 \times 1200$ & 945.3149 & 0.484 & -21.3 & 2.0 & & \\
6 & 19.01 .12 & $3 \times 1200$ & 946.3040 & 0.778 & 100.4 & 1.4 & -304.5 & 6.8 \\
7 & 25.01 .12 & $3 \times 1200$ & 952.3784 & 0.582 & 37.0 & 1.9 & -165.8 & 16.2 \\
8 & 26.01 .12 & $3 \times 1200$ & 953.3117 & 0.860 & 76.0 & 1.8 & -250.3 & 5.7 \\
9 & 31.01 .12 & $3 \times 1200$ & 958.3868 & 0.367 & -119.5 & 1.6 & 191.8 & 11.9 \\
10 & 14.02 .12 & $3 \times 1200$ & 972.2281 & 0.478 & -30.5 & 1.3 & & \\
11 & 14.02 .12 & $3 \times 1200$ & 972.3527 & 0.515 & 1.6 & 1.4 & -54.6 & 16.2 \\
12 & 15.02 .12 & $3 \times 1200$ & 973.2356 & 0.778 & 95.7 & 2.6 & -301.5 & 8.5 \\
13 & 15.02 .12 & $3 \times 1200$ & 973.3800 & 0.821 & 88.7 & 2.6 & -271.9 & 8.5 \\
14 & 20.02 .12 & $3 \times 1200$ & 978.3171 & 0.287 & -144.7 & 1.0 & 246.5 & 6.9 \\
15 & 21.02 .12 & $3 \times 1200$ & 979.2282 & 0.558 & 27.9 & 2.8 & -110.0 & 5.7 \\
16 & 21.02 .12 & $3 \times 1200$ & 979.3019 & 0.580 & 35.4 & 3.6 & -149.8 & 33.1 \\
17 & 21.02 .12 & $3 \times 1200$ & 979.4024 & 0.609 & 61.9 & 3.3 & -200.5 & 23.1 \\
18 & 13.03 .12 & $3 \times 1200$ & 1000.1823 & 0.782 & 94.9 & 1.7 & -312.3 & 6.4 \\
19 & 14.03 .12 & $3 \times 1200$ & 1001.2654 & 0.104 & -102.2 & 2.2 & 165.9 & 10.2 \\
20 & 29.03 .12 & $3 \times 1200$ & 1016.2018 & 0.540 & 8.3 & 4.1 & -91.5 & 9.8 \\
\hline & & & & & & & & \\
& & & & \\
1
\end{tabular}

system was carried out at this observatory, producing one of the most complete light curves [8].

\section{SPECTROSCOPIC OBSERVATIONS AND PRIMARY REDUCTION}

The spectroscopic observations of CC Cas were carried out during the period from March 30, 2011 through March 29, 2012 using the high-resolution fiber-optical echelle spectrometer [9] $(R=30000)$ of the alt-azimuth telescope $(D=1.21 \mathrm{~m}$, $F=12.0 \mathrm{~m})$ [10]. The detector employed was a CCD camera made at the Advanced Design Laboratory of the Special Astrophysical Observatory based on the E2V 40-42 CCD chip $(2048 \times 2048$ px with a pixel size of $13.5 \mu \mathrm{m}$ ) cooled to the liquid-nitrogen temperature by the CRYOTIGER closed-loop system. During the observations, 61 spectral orders covering the wavelength interval 3900-7700 A were recorded simultaneously.

Since this spectroscopic study of CC Cas was one of the first observational programs involving the echelle spectrometer, we opted for the observational scheme with many registrations of the calibrating spectra of a Th-Ar lamp. Each observing run of CC Cas consisting of three successive 20-minute exposures began and ended with the recording of the Th-Ar lamp spectrum. Such an observational scheme made it possible to take into account the possible shifts of the spectrograph wavelength scale relative to the CCD pixels during the subsequent reduction.

To keep the image of the star on the fiber end, the telescope was manually guided during exposure by moving the instrument along both axes at minimum speed. Guiding was controlled on the screen, by monitoring the image produced by the field-of-view 


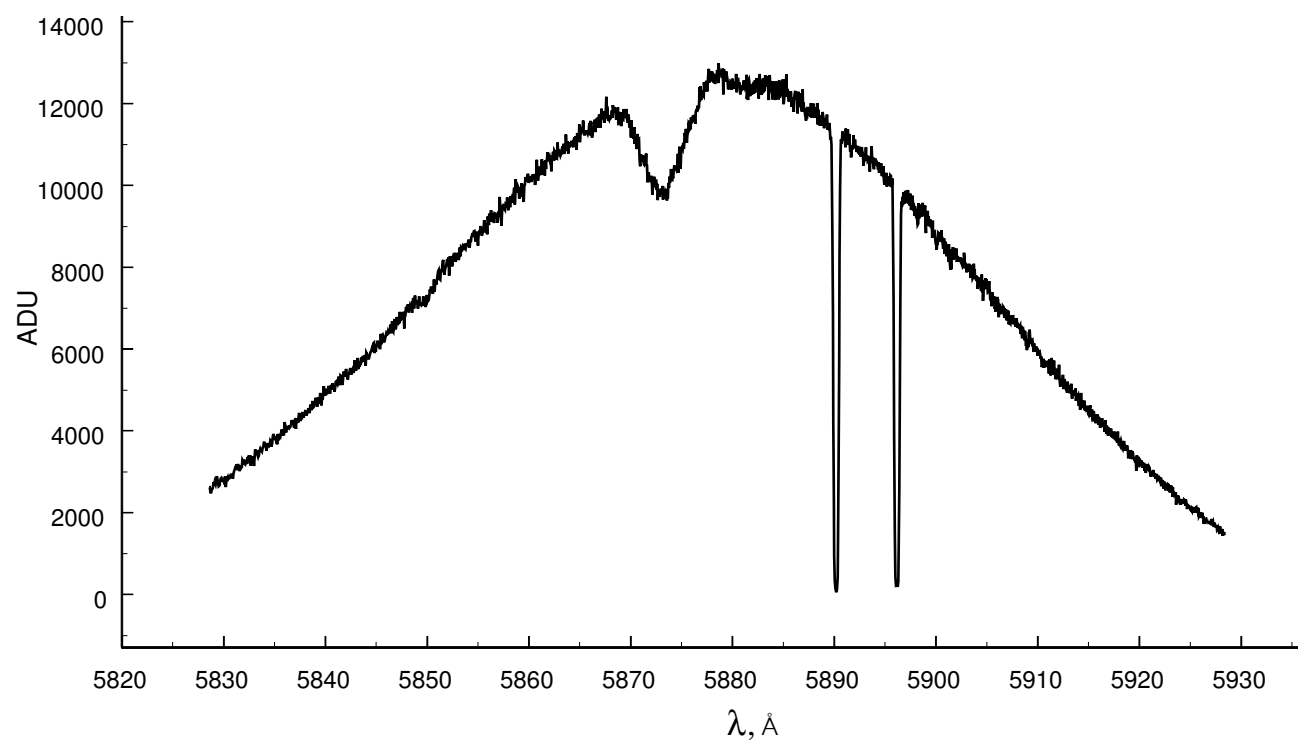

Fig. 1. An extracted spectral order in the vicinity of the He I $\lambda 5876 \AA$ line. The line profile of the secondary component of CC Cas can be clearly seen to the right of the broad line profile of the primary. The two strong narrow lines are the interstellar sodium doublet.
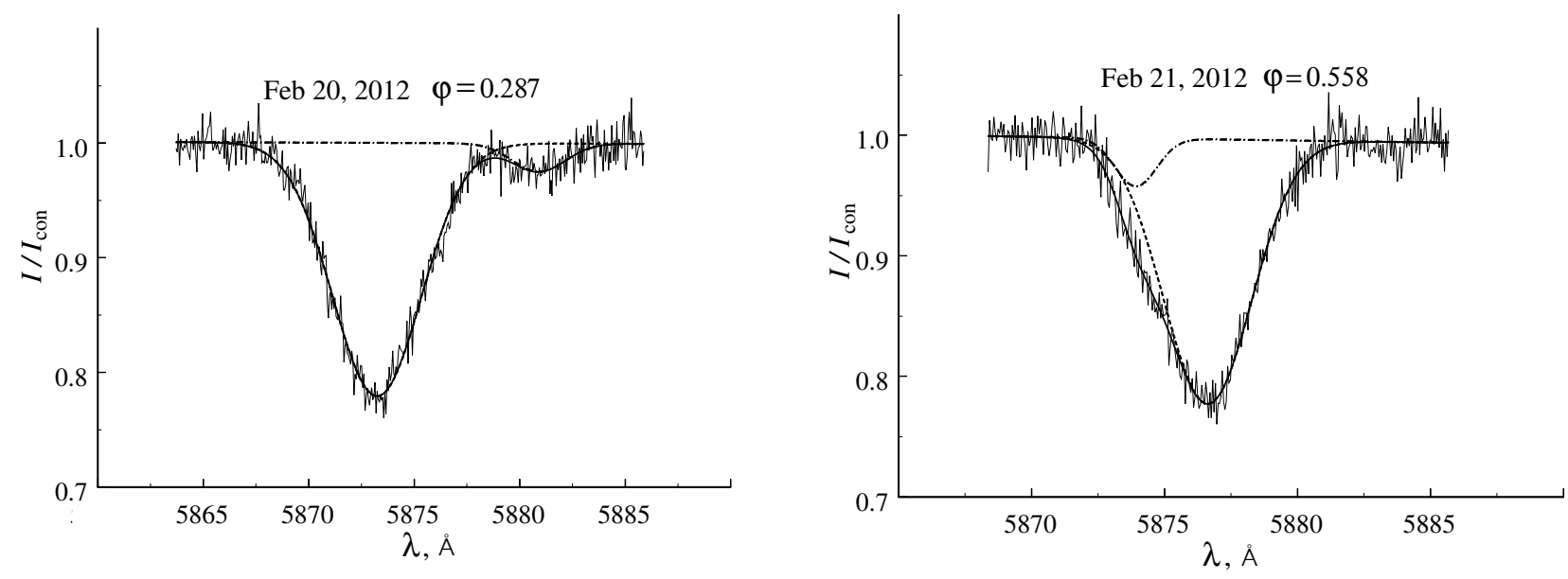

Fig. 2. Bi-gaussian fit of the He I $\lambda 5876 \AA$ line profiles. The solid, dotted, and dashed-and-dotted lines show the total profile, the profile of the primary, and that of the secondary, respectively.

guiding camera mounted on the suspended part of the spectrometer.

During the observations we took a total of $20 \mathrm{spec}-$ tra of CC Cas with an effective exposure of one hour (three 20-minute frames). Given the period of the system, a longer signal integration time would result in an appreciable spread of the spectral lines due to the orbital motion of the components, whereas a shorter integration time would decrease of the signal-to-noise ratio of the spectrum. Table 1 lists the obtained spectra. The listed time of each spectrum is the mid-time of each frame triad.

We used the DECH package [11] to perform the primary reduction of the CCD frames of the echelle spectrum of CC Cas. To remove cosmic ray hits and to increase the signal-to-noise ratio, we performed median averaging of each frame triad followed by the subtraction of the zero-exposure frames (bias) taken

Table 2. Parameters of the radial velocity curves

\begin{tabular}{c|c|c}
\hline & $\begin{array}{c}\text { Primary } \\
\text { component }\end{array}$ & $\begin{array}{c}\text { Secondary } \\
\text { component }\end{array}$ \\
\hline$V_{0}, \mathrm{~km} / \mathrm{s}$ & $-19.8 \pm 1.4$ & $-20.9 \pm 2.9$ \\
$K, \mathrm{~km} / \mathrm{s}$ & $-121.3 \pm 2.2$ & $291.3 \pm 4.5$ \\
\hline
\end{tabular}


Table 3. Summary table of the parameters of CC Cas

\begin{tabular}{l|c|c|c}
\hline Parameter & $\begin{array}{c}\text { Photographic } \\
\text { plate [1, 4] }\end{array}$ & $\begin{array}{c}\text { Reticon } \\
{[4]}\end{array}$ & $\begin{array}{c}\text { CCD } \\
\text { This paper }\end{array}$ \\
\hline$V_{0}, \mathrm{~km} / \mathrm{s}$ & $-9.9 \pm 2.4$ & $-10.4 \pm 2.0$ & $-20.4 \pm 2.1$ \\
$K_{1}, \mathrm{~km} / \mathrm{s}$ & $138.0 \pm 4.0$ & $116.0 \pm 1.8$ & $123.9 \pm 2.0$ \\
$K_{2}, \mathrm{~km} / \mathrm{s}$ & & $285.9 \pm 7.3$ & $292.4 \pm 4.6$ \\
$P$, day & 3.366277 & 3.366324 & 3.366604 \\
$\sigma_{1}, \mathrm{~km} / \mathrm{s}$ & 10.3 & 5.7 & 5.9 \\
$\sigma_{2}, \mathrm{~km} / \mathrm{s}$ & & 27.9 & 11.9 \\
$M_{1} \sin ^{3} i, M_{\odot}$ & $18.3 \pm 1.4$ & $16.1 \pm 0.8$ & $17.5 \pm 1.0$ \\
$M_{2} \sin ^{3} i, M_{\odot}$ & $8.8 \pm 0.8$ & $6.5 \pm 0.4$ & $7.4 \pm 0.5$ \\
$\left(a_{1}+a_{2}\right) \sin i, R_{\odot}$ & $28.3 \pm 0.9$ & $26.2 \pm 0.6$ & $27.0 \pm 0.4$ \\
$q=M_{1} / M_{2}$ & $2.1 \pm 0.1$ & $2.41 \pm 0.04$ & $2.36 \pm 0.05$ \\
\hline
\end{tabular}

on the same date immediately before the observations of the stellar spectra. We also performed pairwise averaging of the frames with the Th-Ar lamp spectra taken before and after each triad of stellar frames. Further reduction included extraction of the orders of the echelle spectrum, construction of the dispersion curves, and determination of the continuum level.

As expected for an O9-B0 type star, the resulting orders of the spectrum of CC Cas exhibit absorption peaks of HeI and Balmer lines, as well as nitrogen, sulphur, and oxygen lines. The strongest line is He I $\lambda 5876 \AA$. The remaining lines have smaller

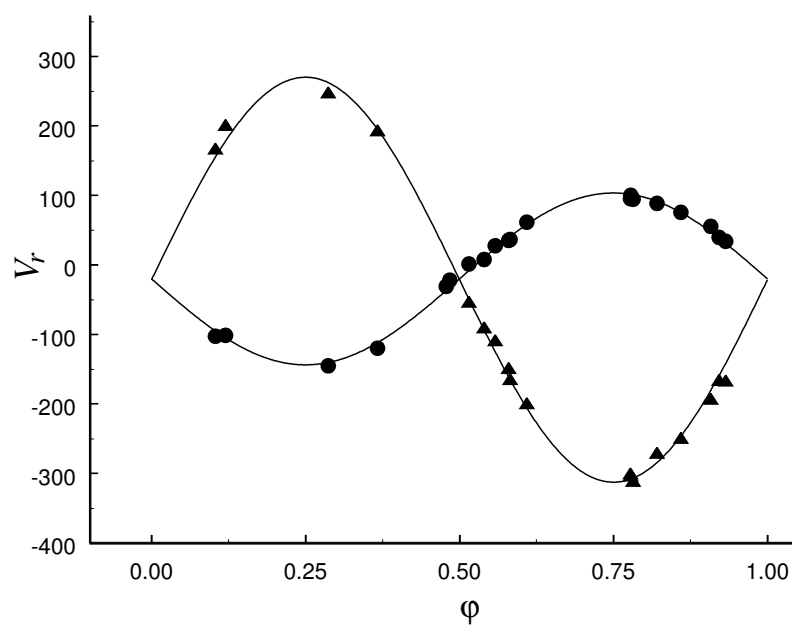

Fig. 3. Radial velocity curves of the components of CC Cas (the solid lines) based on the results of CCD observations. The circles and triangles show the radial velocities of the primary and secondary components, respectively. depths and are more noisy. The signal-to-noise ratio averaged over the spectrum varied from 40 to 80 . As an illustration, we show in Fig. 1 a spectral order in the region of the HeI $\lambda 5876 \AA$ line before its normalization to the continuum.

\section{RADIAL VELOCITY CURVES}

We decided to construct the radial velocity curves based on the He I $\lambda 5876 \AA$ line, because it is the strongest feature in the spectrum of CC Cas. Its profiles taken at phases close to quadrature phases show clear trace signatures of the second component. We decomposed the line blends using the classical method by fitting them to a sum of Gaussians via the Marquardt non-linear least squares procedure. Figure 2 shows examples of the decomposition of the HeI $\lambda 5876 \AA$ line at a close-to-quadrature phase (left) and at the eclipse phase (right), when the profiles of the lines of individual components of CC Cas overlapped considerably. After decomposing the profiles, we determined the radial velocities of both components. For the primary component, the velocities were measured on all of the spectra. We failed to determine the radial velocities for the secondary, fainter component at two close-to-mideclipse phases because of the strong overlap of the profiles. The last four columns in Table 1 list the radial velocities of the components and the formal errors based on the profile fits.

Practically all the photometric studies and the last spectroscopic investigation [4] showed no appreciable orbital eccentricity in CC Cas. We therefore adopted sinusoidal relations for the radial velocity curves. In 
our fits we varied the period of the system, because of the excessive scatter of the phased radial velocity curve based on the ephemeris of Hill et al. [4] and the systematic offset of data points relative to the sinusoidal relations computed with this ephemeris. The new ephemeris based exclusively on our observations has the following form:

$$
\mathrm{JD}_{\odot}\left(I_{\min }\right)=2455647.4234+3.3666045 \mathrm{E} .
$$

The inferred period is longer than that reported by Hill et al. [4], but somewhat shorter than the period determined by Pearce et al. [1] and used by a number of authors including, e.g., [8]. Table 2 lists the inferred $V_{0}, K_{1}$, and $K_{2}$ values, and Fig. 3 shows graphically the resulting sinusoidal fits of the inferred radial velocities of the components of CC Cas.

\section{DISCUSSION OF RESULTS}

It is evident from Fig. 3 that the phased radial velocity curves of both components computed with the ephemeris (1) fit the sinusoidal relations well, and this fact indicates that there is no appreciable eccentricity in their orbits. The resulting $V_{0}$ values are the same for both components within the quoted errors (see Table 2). This fact confirms the validity of the refined period suggesting that it is better than that reported by Hill et al. [4], and provides indirect evidence for a possible process of period variation.

Table 3 lists the parameters of CC Cas that we computed for the average center-of-mass velocity of the system, $V_{0}=\left(V_{0}^{1}+V_{0}^{2}\right) / 2$. For comparison, we also list in the same table the corresponding data from Pearce et al. [1] and Hill et al. [4].

Whereas the inferred $K_{1}$ and $K_{2}$ values differ little from the corresponding estimates obtained by other authors, our $V_{0}$ estimate proved to be greater by $-10 \mathrm{~km} / \mathrm{s}$. Such a large discrepancy may be due both to a real change in the center-of-mass velocity of the system and to some instrumental effect, e.g., a shift of the spectrograph wavelength scale during the observations caused by a temperature variation. To verify the presence of such an effect, we measured the radial velocities of the telluric lines in the $\mathrm{O}_{2} \lambda 6890 \AA$ band and those of the interstellar sodium doublet practically on all the echelle spectra obtained. We list the results of these measurements in Table 4 . The last row of the table lists the radial velocities of the telluric lines and of the interstellar sodium lines averaged over all the spectra. As is evident from these data, the average radial velocities of the telluric lines in the $\mathrm{O}_{2} \lambda 6890 \AA$ band are close to zero and their error does not exceed $\pm 0.5 \mathrm{~km} / \mathrm{s}$. The measurements of the radial velocities of two interstellar sodium lines- Na I $\lambda 5890 \AA$ and Na I $\lambda 5896 \AA$ yielded the same result with the same
Table 4. Radial velocities of the $\mathrm{O}_{2} \lambda 6890 \AA$ band telluric lines and of the interstellar sodium doublet

\begin{tabular}{|c|c|c|c|c|}
\hline $\begin{array}{c}\mathrm{JD}_{\odot} \\
2455000+\end{array}$ & $\begin{array}{c}V_{r}, \mathrm{~km} / \mathrm{s} \\
\mathrm{O}_{2} \\
\lambda 6890 \AA\end{array}$ & $\begin{array}{c}\sigma, \\
\mathrm{km} / \mathrm{s}\end{array}$ & $\begin{array}{c}V_{r}, \mathrm{~km} / \mathrm{s} \\
\mathrm{NaI} \\
\lambda 5890 \AA\end{array}$ & $\begin{array}{c}V_{r}, \mathrm{~km} / \mathrm{s} \\
\mathrm{Na} \mathrm{I} \\
\lambda 5896 \AA\end{array}$ \\
\hline 651.1943 & & & -12.2 & -11.7 \\
\hline 889.5081 & 0.64 & 0.13 & -11.0 & -11.0 \\
\hline 889.5539 & -0.01 & 0.10 & & \\
\hline 889.5913 & -0.77 & 0.31 & & \\
\hline 945.3149 & -0.46 & 0.21 & -11.6 & -11.2 \\
\hline 946.3040 & 0.80 & 0.21 & -10.1 & -9.9 \\
\hline 952.3784 & 0.67 & 0.21 & -10.5 & -10.2 \\
\hline 953.3117 & 0.02 & 0.19 & -10.8 & -10.6 \\
\hline 958.3868 & 0.01 & 0.22 & -10.8 & -10.7 \\
\hline 972.2281 & 0.46 & 0.23 & -11.6 & -11.2 \\
\hline 972.3527 & -0.65 & 0.18 & -10.6 & -10.3 \\
\hline 973.2356 & 0.62 & 0.18 & -10.1 & -10.2 \\
\hline 973.3800 & -0.05 & 0.35 & -10.8 & -10.9 \\
\hline 978.3171 & 0.15 & 0.26 & -10.7 & -10.6 \\
\hline 979.2282 & 0.12 & 0.16 & -11.5 & -11.3 \\
\hline 979.3019 & -0.35 & 0.18 & & \\
\hline 979.4024 & 0.05 & 0.25 & & \\
\hline 1000.1823 & -0.09 & 0.33 & -11.1 & -10.7 \\
\hline 1001.2654 & 0.47 & 0.23 & -10.6 & -10.3 \\
\hline 1016.2018 & 0.51 & 0.20 & -10.6 & -10.4 \\
\hline mean & $\begin{array}{r}0.11 \\
\pm 0.46\end{array}$ & 0.22 & $\begin{array}{c}-10.9 \\
\pm 0.5\end{array}$ & $\begin{array}{l}-10.7 \\
\pm 0.5\end{array}$ \\
\hline
\end{tabular}

accuracy. We found the mean radial velocities of both lines averaged over the entire observing period to differ by no more than $0.2 \mathrm{~km} / \mathrm{s}$, i.e., by less than the measurement error.

The third column in Table 4 lists the standard errors of the measured radial velocities of the narrow telluric lines in individual spectra computed by 30 non-blended $\mathrm{O}_{2}$ lines recorded in the given spectral order. The average standard error of a single measurement is $\pm 0.2 \mathrm{~km} / \mathrm{s}$. We can certify that the radial velocities of interstellar sodium lines have been determined with the same accuracy, since the proce- 
dure of their measurement was identical to that used to measure the telluric lines.

The obtained result indicates the absence (during the entire observing period) of any significant unaccounted for instrumental effect that may have caused a shift of the wavelength scale. Therefore, we can definitely conclude that the $V_{0}$ value obtained in this study is the real center-of-mass velocity of the system at the present epoch as measured from the He I $\lambda 5876 \AA$ line. This result may support the hypothesis of the presence of a third body in the CC Cas system.

It is evident from Table 3 that the standard deviation $\sigma_{2}$ of the measured radial velocities from the radial velocity curve of the secondary component estimated in this study is almost two and a half times smaller than the corresponding value reported by Hill et al. [4].

\section{CONCLUSIONS}

In this part of our study of the eclipsing binary CC Cas with early-type components we used the 20 first-ever high-resolution CCD fiber-optical echelle spectra to measure the radial velocities and obtain the radial velocity curves for both components. These observations allowed us to determine the center-of-mass velocity $V_{0}$ and to refine the orbital period of the system. This is the second study of CC Cas involving the radial velocity measurements of the secondary component, and the scatter of data points on our radial velocity curve proved to be 2.5 times smaller than that in the previous study [4].

The resulting estimates of the period and of the center-of-mass radial velocity differ appreciably from those published in the literature. It can therefore be concluded that the CC Cas system may contain a third body.

\section{ACKNOWLEDGMENTS}

This work was partially supported by the Federal Target Program "Research and Development in Priority Fields of Russian Science and Technology Complex for 2007-2013" (state contract no. 14.518.11.7064).

\section{REFERENCES}

1. J. A. Pearce, Publ. of the Dominion Astrophys. Obs. Victoria 4, 67 (1927).

2. P. Guthnick, R. Prager, Astron. Nachr. 239, 13 (1930).

3. S. Gaposchkin, Annals of Harvard College Obs. 113, 67 (1953).

4. G. Hill, R. W. Hilditch, G. C. L. Aikman, and B. Khalesseh, Astronom. and Astrophys. 282, 455 (1994).

5. P. Mayer, Bull. Astron. Inst. Czechosl. 38, 58 (1987).

6. D. M. Gibson and R. M. Hjellming, Publ. Astronom. Soc. Pacific 86, 652 (1974).

7. I. D. Howarth and R. K. Prinja, Astrophys. J. Suppl. 69, 527 (1989).

8. T. S. Polushina, Peremennye Zvezdy 22, 834 (1988) [in Russian].

9. V. E. Panchuk, M. V. Yushkin, M. V. Yakopov, Astrophysical Bulletin 66, 355 (2011).

10. S. Yu. Gorda, P. E. Zakharova, V. V. Krushinskii, and E. D. Kuznetsov, in Proc. 40th Student Sci. Conf. on Physics of Space (Ural Federal University, Ekaterinburg, 2011), p. 110.

11. G. A. Galazutdinov, Preprint SAO RAS, 92, 1 (1992). 